\title{
Split quaternion matrices
}

Yasemin Alagöz, Kürşat Hakan Oral, and Salim Yüce 


\title{
SPLIT QUATERNION MATRICES
}

\author{
YASEMIN ALAGÖZ, KÜRŞAT HAKAN ORAL, AND SALIM YÜCE
}

Received 7 June, 2011

\begin{abstract}
In this paper, we consider split quaternions and split quaternion matrices. Firstly, we give some properties of split quaternions. After that we investigate split quaternion matrices using properties of complex matrices. Then we define the complex adjoint matrix of split quaternion matrices and we describe some of their properties. Furthermore, we give the definition of $q$-determinant of split quaternion matrices.
\end{abstract}

2000 Mathematics Subject Classification: 11R52; 15A33

Keywords: quaternions, split quaternions, matrices of split quaternions

\section{INTRODUCTION}

Sir William Rowan Hamilton introduced the set of quaternions in 1843, which can be represented as

$$
\mathbf{H}=\left\{a=a_{0}+a_{1} i+a_{2} j+a_{3} k: \quad a_{0}, a_{1}, a_{2}, a_{3} \in \mathbb{R}\right\}
$$

where $i^{2}=j^{2}=k^{2}=-1$ and $i j k=-1$. One can see easily that $i j=-j i=k$. Thus the set of quaternions is a skew-field. Since quaternions are non-commutative, they differs from complex numbers $(\mathbb{C})$ and real numbers $(\mathbb{R})$. For example, for matrices of quaternions the left eigenvalues and the right eigenvalues must be not the same. Because of this, various authors have been studied quaternion matrices, for example [1], [4], [5] and [6]. After the work of Hamilton, in 1849, James Cockle introduced the set of split quaternions, which can be represented as

$$
\mathbf{H}_{\mathbf{s}}=\left\{q=q_{0}+q_{1} i+q_{2} j+q_{3} k: \quad q_{0}, q_{1}, q_{2}, q_{3} \in \mathbb{R}\right\}
$$

where $i^{2}=-1, j^{2}=k^{2}=1$ and $i j k=1$. This set is 4-dimensional like the set of quaternions. $\mathbf{H}_{\mathrm{s}}$ is noncommutative, too. But $\mathbf{H}_{\mathrm{s}}$ contains zero-divisors, nilpotent elements and nontrivial idempotents. For more properties of split quatenions the reader is referred to [2] and [3].

In this paper, we will investigate split quaternion matrices. Firstly in Section 2, we will give some properties of split quaternions which will be used in the following sections. After that, in Section 3, we will introduce split quaternion matrices and give some properties of them. Finally in Section 4, we define the complex adjoint matrix 
of split quaternion matrices and we give some properties of these matrices. Then we will define the determinant of a split quaternion matrices using its adjoint matrix.

\section{SPLIT QUATERNIONS}

A split quaternion $q$ is a vector of the form

$$
q=a_{0}+a_{1} i+a_{2} j+a_{3} k
$$

where $a_{0}, a_{1}, a_{2}, a_{3}$ are real numbers. Split quaternion algebra is an associative, non-commutative, non-division ring with four basis elements $\{1, i, j, k\}$ satisfying the equalities

$$
\begin{aligned}
& i^{2}=-1, j^{2}=k^{2}=1, \\
& i j=-j i=k, j k=-k j=-i, k i=-i k=j .
\end{aligned}
$$

Let us denote the algebra of split quaternions by $\mathbf{H}_{S}$. For any $q=a_{0}+a_{1} i+a_{2} j+$ $a_{3} k \in \mathbf{H}_{S}$ we define $\operatorname{Re} q=a_{0}$, the real part of $q ; \operatorname{Im} q=a_{1} i+a_{2} j+a_{3} k$, the imaginary part of $q$. The conjugate of a split quaternion is denoted by $q^{*}=\bar{q}$ and it is

$$
q^{*}=\bar{q}=a_{0}-a_{1} i-a_{2} j-a_{3} k
$$

The norm of a split quaternion is defined as

$$
\|q\|=\sqrt{\left|q q^{*}\right|}=\sqrt{\left|I_{q}\right|}
$$

where $I_{q}=q q^{*}=a_{0}^{2}+a_{1}^{2}-a_{2}^{2}-a_{3}^{2}$.If $\|q\|=1$ then $q$ is called unit split quaternion. Note that $p=\frac{q}{\|q\|}$ is a unit split quaternion for $q \in \mathbf{H}_{S}$ with $\|q\| \neq 0$. The inverse of the split quaternion $q$ is

$$
q^{-1}=\frac{q^{*}}{I_{q}}, \text { if } I_{q} \neq 0
$$

Theorem 1. Split quaternions satisfy the following properties for the elements $p, q$ and $r \in \mathbf{H}_{S}$.

(i) $q^{*} q=q q^{*}$,

(ii) $j c=\bar{c} j$ or $j c j^{*}=\bar{c}$ for any complex number $c$,

(iii) $q^{2}=|\operatorname{Re} q|^{2}-\|\operatorname{Im} q\|^{2}+2 \operatorname{Re} q \operatorname{Im} q$,

(iv) $(q p)^{*}=p^{*} q^{*}$

(v) $(p q) r=p(q r)$,

(vi) $p q \neq q p$ in general,

(vii) $q^{*}=q$ if and only if $q$ is a real number,

(viii) $\frac{q^{*}}{I_{q}}$ is the inverse of $q$ if $a_{0}^{2}+a_{1}^{2} \neq a_{2}^{2}+a_{3}^{2}$,

(ix) Every split quaternion $q$ can be uniquely expressed as $q=c_{1}+c_{2} j$, where $c_{1}$ and $c_{2}$ are complex numbers.

Theorem 2. Every split quaternion can be represented by a $2 \times 2$ complex matrix. 
Proof. Let $q \in \mathbf{H}_{S}$, then there exist complex numbers $a_{1}$ and $a_{2}$ such that $q=a_{1}+$ $a_{2} j$ by Theorem 1(ix). The linear map $f_{q}: \mathbf{H}_{S} \rightarrow \mathbf{H}_{S}$ is defined by $f_{q}(p)=p q$ for all $p \in \mathbf{H}_{S}$. This map is bijective and

$$
\begin{aligned}
& f_{q}(1)=1\left(a_{1}+a_{2} j\right)=a_{1}+a_{2} j \\
& f_{q}(j)=j\left(a_{1}+a_{2} j\right)=\overline{a_{2}}+\overline{a_{1}} j .
\end{aligned}
$$

With this transformation split quaternions are defined as subset of the matrix ring $M_{2}(\mathbb{C})$, the set of $2 \times 2$ complex matrices:

$$
\mathbf{H}_{S}^{\prime}=\left\{\left(\begin{array}{cc}
a_{1} & a_{2} \\
\overline{a_{2}} & \overline{a_{1}}
\end{array}\right) ; a_{1}, a_{2} \in \mathbb{C}\right\} .
$$

$\mathbf{H}_{S}$ and $\mathbf{H}_{S}^{\prime}$ are essentially the same.

Note that,

$$
\mathcal{M}: q=a_{1}+a_{2} j \in \mathbf{H}_{S} \rightarrow q^{\prime}=\left(\begin{array}{cc}
a_{1} & a_{2} \\
\overline{a_{2}} & \overline{a_{1}}
\end{array}\right) \in \mathbf{H}_{S}^{\prime}
$$

is bijective and preserves the operations. Furthermore $\|q\|^{2}=\operatorname{det} q^{\prime}$.

\section{SPLIT QUATERNION MATRICES}

The set $M_{m \times n}\left(\mathbf{H}_{S}\right)$ denotes all $m \times n$ type matrices with split quaternion entries. If $m=n$, then the set of split quaternion matrices is denoted by $M_{n}\left(\mathbf{H}_{S}\right)$. The ordinary matrix addition and multiplication are defined. With these operations $M_{n}\left(\mathbf{H}_{S}\right)$ is a ring with a unit. The left (right) scalar multiplication is defined as, for $A=\left(a_{s t}\right) \in$ $M_{m \times n}\left(\mathbf{H}_{S}\right), q \in \mathbf{H}_{S}$,

$$
q A=\left(q a_{s t}\right) \text { and } A q=\left(a_{s t} q\right) .
$$

Here for $B \in M_{n \times o}\left(\mathbf{H}_{S}\right), p \in \mathbf{H}_{S}$ we obtain

$$
\begin{aligned}
& q(A B)=(q A) B, \\
& (A q) B=A(q B), \\
& (p q) A=p(q A) .
\end{aligned}
$$

$M_{m \times n}\left(\mathbf{H}_{S}\right)$ is a left (right) vector space over $\mathbf{H}_{S}$.

For $A=\left(a_{s t}\right) \in M_{m \times n}\left(\mathbf{H}_{S}\right), \bar{A}=\left(\overline{a_{s t}}\right)=\left(a_{s t}^{*}\right) \in M_{m \times n}\left(\mathbf{H}_{S}\right)$ is the conjugate of $A ; A^{T}=\left(a_{t s}\right) \in M_{n \times m}\left(\mathbf{H}_{S}\right)$ is the transpose of $A ; A^{*}=(\bar{A})^{T} \in M_{n \times m}\left(\mathbf{H}_{S}\right)$ is the conjugate transpose of $A$.

For a square matrix $A \in M_{n}\left(\mathbf{H}_{S}\right)$ if $A A^{*}=A^{*} A$ then $A$ is called a normal matrix; if $A=A^{*}$ then $A$ is called a Hermitian matrix; if $A A^{*}=I$ then $A$ is called a unitary matrix; for $B \in M_{n}\left(\mathbf{H}_{S}\right)$ if $A B=B A=I$ then $A$ is an invertible matrix. 
Example 1. Let $A=\left(\begin{array}{cc}i & j \\ 0 & k\end{array}\right), B=\left(\begin{array}{cc}i & k \\ 0 & j\end{array}\right) \in M_{2}\left(\mathbf{H}_{S}\right)$. Then,

(i) $A^{-1}=\left(\begin{array}{cc}-i & 1 \\ 0 & k\end{array}\right)$ and $\bar{A}=\left(\begin{array}{cc}-i & -j \\ 0 & -k\end{array}\right)$

(ii) $(\bar{A})^{-1}=\left(\begin{array}{cc}i & -1 \\ 0 & -k\end{array}\right) \neq\left(\begin{array}{cc}i & 1 \\ 0 & -k\end{array}\right)=\overline{\left(A^{-1}\right)}$

(iii) $\left(A^{T}\right)^{-1}=\left(\begin{array}{cc}-i & 0 \\ -1 & k\end{array}\right) \neq\left(\begin{array}{cc}-i & 0 \\ 1 & k\end{array}\right)=\left(A^{-1}\right)^{T}$

(iv) $\overline{A B}=\left(\begin{array}{cc}-1 & j+1 \\ 0 & -i\end{array}\right) \neq\left(\begin{array}{cc}-1 & -j+1 \\ 0 & i\end{array}\right)=\bar{A} \bar{B}$

(v) $(A B)^{T}=\left(\begin{array}{cc}-1 & 0 \\ -j+1 & i\end{array}\right) \neq\left(\begin{array}{cc}-1 & 0 \\ j+1 & -i\end{array}\right)=B^{T} A^{T}$

With these examples we get the following Corollary for split quaternion matrices.

Corollary 1. Let $A \in M_{m \times n}\left(\mathbf{H}_{S}\right)$ and $B \in M_{n \times o}\left(\mathbf{H}_{S}\right)$. Then the followings are satisfied:

(i) $(\bar{A})^{-1} \neq \overline{\left(A^{-1}\right)}$ in general;

(ii) $\left(A^{T}\right)^{-1} \neq\left(A^{-1}\right)^{T}$ in general;

(iii) $\overline{A B} \neq \bar{A} \bar{B}$ in general;

(iv) $(A B)^{T} \neq B^{T} A^{T}$ in general.

Theorem 3. Let $A \in M_{m \times n}\left(\mathbf{H}_{S}\right)$ and $B \in M_{n \times o}\left(\mathbf{H}_{S}\right)$. Then,

(i) $(\bar{A})^{T}=\overline{\left(A^{T}\right)}$,

(ii) $(A B)^{*}=B^{*} A^{*}$,

(iii) $(A B)^{-1}=B^{-1} A^{-1}$ if $A$ and $B$ are invertible,

(iv) $\left(A^{*}\right)^{-1}=\left(A^{-1}\right)^{*}$ if $A$ is invertible.

Proof. (i), (i ii $)$ and (iv) can be easily shown. Now we will prove (ii).

(ii) Let $A=A_{1}+A_{2} j$ and $B=B_{1}+B_{2} j$, where $A_{1}, A_{2}, B_{1}$ and $B_{2}$ are complex matrices. Then

$$
\begin{aligned}
(A B)^{*} & =\left[\left(A_{1} B_{1}+A_{2} \overline{B_{2}}\right)+\left(A_{1} B_{2}+A_{2} \overline{B_{1}}\right) j\right]^{*} \\
& =\left[\left(\overline{A_{1} B_{1}}+\overline{A_{2}} B_{2}\right)-\left(A_{1} B_{2}+A_{2} \overline{B_{1}}\right) j\right]^{T} \\
& =\left(\overline{A_{1} B_{1}}+\overline{A_{2}} B_{2}\right)^{T}-\left(A_{1} B_{2}+A_{2} \overline{B_{1}}\right)^{T} j \\
& =\left(\overline{B_{1}}\right)^{T}\left(\overline{A_{1}}\right)^{T}+\left(B_{2}\right)^{T}\left(\overline{A_{2}}\right)^{T}-\left(B_{2}\right)^{T}\left(A_{1}\right)^{T} j-\left(\overline{B_{1}}\right)^{T}\left(A_{2}\right)^{T} j .
\end{aligned}
$$

On the other hand, for

$$
B^{*}=\left(\overline{B_{1}}\right)^{T}-\left(B_{2}\right)^{T} j \text { and } A^{*}=\left(\overline{A_{1}}\right)^{T}-\left(A_{2}\right)^{T} j
$$


we can obtain

$$
\begin{aligned}
B^{*} A^{*} & =\left(\left(\overline{B_{1}}\right)^{T}-\left(B_{2}\right)^{T} j\right)\left(\left(\overline{A_{1}}\right)^{T}-\left(A_{2}\right)^{T} j\right) \\
& =\left(\overline{B_{1}}\right)^{T}\left(\overline{A_{1}}\right)^{T}+\left(B_{2}\right)^{T}\left(\overline{A_{2}}\right)^{T}-\left(B_{2}\right)^{T}\left(A_{1}\right)^{T} j-\left(\overline{B_{1}}\right)^{T}\left(A_{2}\right)^{T} j .
\end{aligned}
$$

Thus we get

$$
(A B)^{*}=B^{*} A^{*}
$$

Proposition 1. Let $A, B \in M_{n}\left(\mathbf{H}_{S}\right)$, if $A B=I$ then $B A=I$.

Proof. It is well known that this proposition is true for complex matrices. Now let $A=A_{1}+A_{2} j$ and $B=B_{1}+B_{2} j$, where $A_{1}, A_{2}, B_{1}$ and $B_{2}$ are $n \times n$ complex matrices. Then

$$
A B=\left(A_{1} B_{1}+A_{2} \overline{B_{2}}\right)+\left(A_{1} B_{2}+A_{2} \overline{B_{1}}\right) j=I .
$$

From this we get

$$
\left(A_{1}, A_{2}\right)\left(\frac{B_{1}}{B_{2}} \frac{B_{2}}{B_{1}}\right)=(I, 0)
$$

so

$$
\left(\begin{array}{ll}
\frac{A_{1}}{A_{2}} & \frac{A_{2}}{A_{1}}
\end{array}\right)\left(\begin{array}{ll}
\frac{B_{1}}{B_{2}} & \frac{B_{2}}{B_{1}}
\end{array}\right)=\left(\begin{array}{ll}
I & 0 \\
0 & I
\end{array}\right) .
$$

Since $\left(\frac{A_{1}}{A_{2}} \frac{A_{2}}{A_{1}}\right)$ and $\left(\frac{B_{1}}{B_{2}} \frac{B_{2}}{B_{1}}\right)$ are $2 n \times 2 n$ complex matrices, we get

$$
\left(\begin{array}{ll}
\frac{B_{1}}{B_{2}} & \frac{B_{2}}{B_{1}}
\end{array}\right)\left(\begin{array}{ll}
\frac{A_{1}}{A_{2}} & \frac{A_{2}}{A_{1}}
\end{array}\right)=\left(\begin{array}{cc}
I & 0 \\
0 & I
\end{array}\right) .
$$

Then we can write

$$
B_{1} A_{1}+B_{2} \overline{A_{2}}=I, \quad B_{1} A_{2}+B_{2} \overline{A_{1}}=0,
$$

so

$$
\left(B_{1} A_{1}+B_{2} \overline{A_{2}}\right)+\left(B_{1} A_{2}+B_{2} \overline{A_{1}}\right) j=I .
$$

Thus we obtai,

$$
B A=I .
$$




\section{THE COMPLEX ADJOINT MATRIX OF THE SPLIT QUATERNION MATRICES}

In this section we will define the complex adjoint matrix of a split quaternion matrix. After that we will give some relations between split quaternion matrices and their complex adjoint matrices.

Definition 1. Let $A=A_{1}+A_{2} j \in M_{n}\left(\mathbf{H}_{S}\right)$ where $A_{1}$ and $A_{2}$ are complex matrices. We define the $2 n \times 2 n$ complex matrices $\chi_{A}$ as

$$
\left(\begin{array}{ll}
\frac{A_{1}}{A_{2}} & \frac{A_{2}}{A_{1}}
\end{array}\right) .
$$

This matrix $\chi_{A}$ is called the complex adjoint (or adjoint) matrix of the split quaternion matrix $A$.

Example 2. Let $A=\left(\begin{array}{cc}1 & i+j+k \\ 0 & 1\end{array}\right)$ be a split quaternion matrix. Then the complex adjoint matrix of $A$ is

$$
\chi_{A}=\left(\begin{array}{cccc}
1 & i & 0 & 1+i \\
0 & 1 & 0 & 0 \\
0 & 1-i & 1 & -i \\
0 & 0 & 0 & 1
\end{array}\right)
$$

Furthermore, $A^{*}=\left(\begin{array}{cc}1 & 0 \\ -i-j-k & 1\end{array}\right)$ and the complex adjoint matrix of $A^{*}$ is

$$
\chi_{\left(A^{*}\right)}=\left(\begin{array}{cccc}
1 & 0 & 0 & 0 \\
-i & 1 & -1-i & 0 \\
0 & 0 & 1 & 0 \\
-1+i & 0 & i & 1
\end{array}\right)
$$

But

$$
\left(\chi_{A}\right)^{*}=\left(\begin{array}{llll}
1 & 0 & 0 & 0 \\
-i & 1 & 1+i & 0 \\
0 & 0 & 1 & 0 \\
1-i & 0 & i & 1
\end{array}\right) .
$$

Thus, we get $\chi_{\left(A^{*}\right)} \neq\left(\chi_{A}\right)^{*}$.

Theorem 4. Let $A, B \in M_{n}\left(\mathbf{H}_{S}\right)$. Then the followings hold

(i) $\chi_{I_{n}}=I_{2 n}$;

(ii) $\chi_{A+B}=\chi_{A}+\chi_{B}$;

(iii) $\chi_{A B}=\chi_{A} \chi_{B}$;

(iv) $\chi_{A^{-1}}=\left(\chi_{A}\right)^{-1}$ if $A^{-1}$ exist;

(v) $\chi_{A^{*}} \neq\left(\chi_{A}\right)^{*}$ in general. 
Proof. (i), (ii) and (iv) can be easily shown. For (v), we have given Example 2. Now we will prove (iii).

(i i i ) Let $A, B \in M_{n}\left(\mathbf{H}_{S}\right)$ where $A=A_{1}+A_{2} j$ and $B=B_{1}+B_{2} j$ for the complex matrices $A_{1}, A_{2}, B_{1}$ and $B_{2}$. Then the complex adjoint matrices of $A$ and $B$ are

$$
\chi_{A}=\left(\frac{A_{1}}{A_{2}} \frac{A_{2}}{A_{1}}\right), \quad \chi_{B}=\left(\begin{array}{ll}
\frac{B_{1}}{B_{2}} & \frac{B_{2}}{B_{1}}
\end{array}\right) .
$$

If we calculate $A B$ for $A=A_{1}+A_{2} j$ and $B=B_{1}+B_{2} j$, then we have

$$
A B=\left(A_{1} B_{1}+A_{2} \overline{B_{2}}\right)+\left(A_{1} B_{2}+A_{2} \overline{B_{1}}\right) j .
$$

So for the complex adjoint matrix of $A B$ we can write

$$
\chi_{A B}=\left(\begin{array}{cc}
A_{1} B_{1}+A_{2} \overline{B_{2}} & A_{1} B_{2}+A_{2} \overline{B_{1}} \\
\overline{A_{1} B_{2}}+\overline{A_{2} B_{1}} & \overline{A_{1} B_{1}}+\overline{A_{2}} B_{2}
\end{array}\right) .
$$

On the other hand,

$$
\chi_{A} \chi_{B}=\left(\begin{array}{ll}
\frac{A_{1}}{A_{2}} & \overline{A_{2}} \\
\overline{A_{1}}
\end{array}\right)\left(\begin{array}{cc}
\frac{B_{1}}{B_{2}} & \overline{B_{2}} \\
\overline{B_{1}}
\end{array}\right)=\left(\begin{array}{cc}
A_{1} B_{1}+\overline{A_{2}} \overline{B_{2}} & \frac{A_{1} B_{2}}{\overline{A_{1} B_{2}}}+\overline{A_{2}} B_{1} \overline{A_{2}} \overline{B_{1}} \\
A_{1} B_{1} & \overline{A_{2}} B_{2}
\end{array}\right) .
$$

Thus we get

$$
\chi_{A B}=\chi_{A} \chi_{B}
$$

Definition 2. Let $A \in M_{n}\left(\mathbf{H}_{S}\right)$ and $\lambda \in \mathbf{H}_{S}$. If $\lambda$ satisfies the equation of $A x=$ $\lambda x(A x=x \lambda)$ then $\lambda$ is called the left (right) eigenvalue of $A$. The set of the left eigenvalue is defined as $\sigma_{l}(A)=\left\{\lambda \in \mathbf{H}_{S}: A x=\lambda x\right.$, for some $\left.x \neq 0\right\}$. This set is called the left spectrum of $A$. Similarly, we define the right spectrum of $A$ as $\sigma_{r}(A)=\left\{\lambda \in \mathbf{H}_{S}: A x=x \lambda\right.$, for some $\left.x \neq 0\right\}$.

Example 3. For the split quaternion matrix,

$$
A=\left(\begin{array}{ll}
0 & i \\
-i & 0
\end{array}\right)
$$

some of the left eigenvalues of $A$ are 1 and -1 . Some of the right eigenvalues of $A$ are $\{ \pm 1, \pm j, \pm k\}$.

Example 4. For the split quaternion matrix,

$$
A=\left(\begin{array}{ll}
0 & j \\
-j & 0
\end{array}\right)
$$

some of the left eigenvalues of $A$ are $\{ \pm k\}$ but none of them is a right eigenvalue. Similarly, some of the right eigenvalues of $A$ are $\{ \pm i\}$. 
Theorem 5. Let $A \in M_{n}\left(\mathbf{H}_{S}\right)$. The followings are equivalent:

(i) $A$ is invertible,

(ii) $A x=0$ has a unique solution 0 ,

(iii) $\left|\chi_{A}\right| \neq 0$, i.e., $\chi_{A}$ is an invertible,

(iv) A has no zero eigenvalue. More precisely if $A x=\lambda x$ or $A x=x \lambda$ for some split quaternion $\lambda$ and some split quaternion vector $x \neq 0$, then $\lambda \neq 0$.

Proof. $(i) \Rightarrow$ (ii) This part is trivial.

(ii ) $\Rightarrow$ (ii i ) Let $A=A_{1}+A_{2} j, x=x_{1}+x_{2} j$ where $A_{1}, A_{2}$ are complex matrices and $x_{1}, x_{2}$ are complex column vectors. Then

$$
A x=\left(A_{1} x_{1}+A_{2} \overline{x_{2}}\right)+\left(A_{1} x_{2}+A_{2} \overline{x_{1}}\right) j .
$$

From $A x=0$ we can write

and

$$
A_{1} x_{1}+A_{2} \overline{x_{2}}=0
$$

$$
A_{1} x_{2}+A_{2} \overline{x_{1}}=0 .
$$

These equations give us the equations

$$
A_{1} x_{1}+A_{2} \overline{x_{2}}=0
$$

and

$$
\overline{A_{2}} x_{1}+\overline{A_{1}} \overline{x_{2}}=0
$$

So we get that

$$
A x=0 \text { if and only if }\left(\frac{A_{1}}{A_{2}} \frac{A_{2}}{A_{1}}\right)\left(\begin{array}{l}
x_{1} \\
\overline{x_{2}}
\end{array}\right)=0 .
$$

That is $\chi_{A}\left(x_{1}, \overline{x_{2}}\right)^{T}=0$. Since $A x=0$ has an unique solution, $\chi_{A}\left(x_{1}, \overline{x_{2}}\right)^{T}=0$ has an unique solution. Thus, since $\chi_{A}$ is a complex matrix, $\chi_{A}$ is invertible.

(ii) $\Leftrightarrow$ (iv) Let $A x=0$ has a unique solution 0 for $A \in M_{n}\left(\mathbf{H}_{S}\right)$. Suppose that $A$ has a zero eigenvalue. Then for some split quaternion vector $x \neq 0$ the equation $A x=\lambda x$ has zero eigenvalue. Thus $A x=0$, and so by our assumption $x=0$, this is a contradiction. Now suppose that $A$ has no zero eigenvalue. If we have $A x=0=\lambda x$, then by our assumption $x=0$.

( $i$ i i $) \Rightarrow$ (i) If $\chi_{A}$ is invertible, then for $A=A_{1}+A_{2} j$ there exist a complex matrix $\left(\begin{array}{ll}B_{1} & B_{2} \\ B_{3} & B_{4}\end{array}\right)$ such that

$$
\left(\begin{array}{ll}
B_{1} & B_{2} \\
B_{3} & B_{4}
\end{array}\right)\left(\begin{array}{ll}
\frac{A_{1}}{A_{2}} & \frac{A_{2}}{A_{1}}
\end{array}\right)=\left(\begin{array}{cc}
I & 0 \\
0 & I
\end{array}\right)
$$

Here we get

$$
B_{1} A_{1}+B_{2} \overline{A_{2}}=I
$$

and

$$
B_{1} A_{2}+B_{2} \overline{A_{1}}=0
$$


Using this equations we can write

$$
\left(B_{1} A_{1}+B_{2} \overline{A_{2}}\right)+\left(B_{1} A_{2}+B_{2} \overline{A_{1}}\right) j=I .
$$

That is

$$
B A=I
$$

for $B=B_{1}+B_{2} j$. So $A$ is an invertible split quaternion matrix by Proposition 1 .

Definition 3. Let $A \in M_{n}\left(\mathbf{H}_{S}\right)$ and $\chi_{A}$ be the complex adjoint matrix of $A$. We define the $q$-determinant of $A$ by $|A|_{q}=\left|\chi_{A}\right|$. Here $\left|\chi_{A}\right|$ is the usual determinant of $\chi_{A}$.

Theorem 6. Let $A, B \in M_{n}\left(\mathbf{H}_{S}\right)$, then

(i) $A$ is invertible $\Leftrightarrow|A|_{q} \neq 0$.

(ii) $|A B|_{q}=|A|_{q}|B|_{q}$, consequently $\left|A^{-1}\right|_{q}=|A|_{q}^{-1}$ if $A^{-1}$ is exist.

(i i i ) $|P A Q|_{q}=|A|_{q}$, for any elementary matrices $P$ and $Q$.

(iv) Cayley-Hamilton Theorem: Let $A$ be a square matrix of split quaternions, and let $F_{A}(\lambda)=\left|\lambda I_{2 n}-\chi_{A}\right|$, which is called the characteristic polynomial of $A$, where $\lambda$ is a complex indeterminant. Then $F_{A}(A)=0$, and $F_{A}\left(\lambda_{0}\right)=0$ if and only if $\lambda_{0}$ is an eigenvalue.

Proof. (i) It is clear from Theorem 5.

(ii) From Theorem 4 we have $\chi_{A B}=\chi_{A} \chi_{B}$. And so $|A B|_{q}=\left|\chi_{A B}\right|=\left|\chi_{A} \chi_{B}\right|=$ $\left|\chi_{A}\right|\left|\chi_{B}\right|=|A|_{q}|B|_{q}$. Here, if we let $B=A^{-1}$ then we can find easily $\left|A^{-1}\right|_{q}=$ $|A|_{q}^{-1}$.

(i i i ) It is sufficient to observe that $\left|\chi_{p}\right|=1$ where $\mathrm{P}$ is an elementary split quaternion matrix.

(iv) $F_{A}(\lambda)$ is a real coefficient polynomial by [5, Corollary 5.1]. For any real coefficient polynomial $f, \chi_{f(A)}=F_{A}\left(\chi_{A}\right)$. Since $\chi_{A}$ is a complex matrix, by the Cayley- Hamilton theorem for complex matrices we have $F_{A}\left(\chi_{A}\right)=0$. Thus we get $\chi_{f(A)}=F_{A}\left(\chi_{A}\right)=0$, hence $F_{A}(A)=0$, as desired.

The rest of this part follows, since every split quaternion is similar to a complex number, and $A x=x \lambda$ is equivalent to $\chi_{A}\left(x_{1},-\overline{x_{2}}\right)^{T}=\lambda\left(x_{1},-\overline{x_{2}}\right)^{T}$, where $x=$ $x_{1}+x_{2} j$ and $\lambda$ is a complex number.

\section{REFERENCES}

[1] S. De Leo, G. Scolarici, and L. Solombrino, "Quaternionic eigenvalue problem," J. Math. Phys., vol. 43, no. 11, pp. 5815-5829, 2002.

[2] L. Kula and Y. Yayli, "Split quaternions and rotations in semi Euclidean space $\mathbb{E}_{2}^{4}$," J. Korean Math. Soc., vol. 44, no. 6, pp. 1313-1327, 2007.

[3] M. Özdemir, "The roots of a split quaternion," Appl. Math. Lett., vol. 22, no. 2, pp. 258-263, 2009.

[4] Q.-W. Wang, H.-S. Zhang, and S.-W. Yu, "On solutions to the quaternion matrix equation $A X B+$ CYD = E," Electron. J. Linear Algebra, vol. 17, pp. 343-358, 2008. 
[5] F. Zhang, "Quaternions and matrices of quaternions," Linear Algebra Appl., vol. 251, pp. 21-57, 1997.

[6] F. Zhang and Y. Wei, "Jordan canonical form of a partitioned complex matrix and its application to real quaternion matrices," Commun. Algebra, vol. 29, no. 6, pp. 2363-2375, 2001.

Authors' addresses

\section{Yasemin Alagöz}

Y1ldız Technical University, Department of Mathematics, 34720, Istanbul, TURKEY

E-mail address: ygulluk@yildiz.edu.tr

\section{Kürşat Hakan Oral}

Yıldız Technical University, Department of Mathematics, 34720, Istanbul, TURKEY

E-mail address: khoral@yildiz.edu.tr

Salim Yüce

Y1ldız Technical University, Department of Mathematics, 34720, Istanbul, TURKEY

E-mail address: sayuce@yildiz.edu.tr 